International Journal of Multidisciplinary Research AND ANALysis

ISSN[Online] : 2643-9875 || ISSN[Print] : 2643-9840

Volume 3 Issue 09 September 2020

DOI: 10.47191/ijmra/v3-i9-03, Impact Factor : 5.522

Page No : $90-93$

\title{
Planning the process of physical preparation at the different stages of the annual training of 17-19 year old girls on the block method
}

\author{
Nodirbek Mahmudovich Yusupov \\ Department "Theory and methodology of football", Uzbek state University of Physical Culture and Sports
}

\begin{abstract}
The article presents new approaches to the structure of the annual training cycle of female players, which are related to the precise quantitative distribution of training in different directions on the me so and micro-cycles. From this perspective, rational planning is concerned with the effects of unidirectional physical loads on the body, both during a single micro-cycle ("unidirectional load micro block") and throughout the entire me so-cycle, with subsequent changes in training stimuli. The use of the block method implies a transition to a new system of regulation of physical loads, which will take the whole process of training 17-19-year-old girls to a new qualitatively higher level.
\end{abstract}

\section{KEYWORDS}

The block method, technical-tactical, teen-player girls, micro-cycle, me so-cycle, specialized loads.

\section{INTRODUCTION}

Achieving a higher level of special physical training by young players is one of the primary tasks of training skilled reserves for professional football / 1,3,5,8 /.Typically, the solution to this problem in sports practice is to further increase the volume and intensity of training loads.Studies $(3,6,7)$ have shown that the amount of work that 17-19 year old players do is significant, and the resources and capabilities of the loco motor system are also limited. Any "early" (inappropriate and unreasonable) acceleration can lead to injury and over-training of the young player. Therefore, the intensification of the training process of adolescent girls requires careful planning, which is associated with a clear distribution of loads in different directions over time at all stages of preparation and the ability to combine them in an optimal manner. It is known that the traditional periodicity of the training process is associated with a comprehensive solution to the problem of improving sports uniforms for successful participation in current competitions. However, practice shows that such an approach to the issue does not provide a level of specialized physical performance ability suitable for young players to play in professional teams. This, in turn, creates the need to develop more effective training programs that allow for the rational planning of physical activity.

\section{METHODOLOGY}

We have previously shown that in the three-year development of block-method training of specific physical performance skills of adolescent girls, each annual training cycle has a certain priority, which is carried out within the developing me so cycles. In this approach, the annual training cycle consists of a continuous process, which consists of three sixteen-week training blocks (TB-1, TB-2, TB-3).The training blocks consist of three me so cycles that differ both in the orientation ("A" -aerobic, "B" glycolytic, "S" -alactate) and in the total value of the physical load-developer - DMSCs and two helpers - AMSCs. Within each such block, the tasks of development of basic physical qualities DMSCs-6 weeks or improvement (HMSCs - 5 weeks), which form a special performance system of female players on the appropriate me so cycles, are solved in sequence. The sequence of application of mesocyclesin different directions ("A", "B", "C") in the training blocks is determined in accordance with the principle of sports training on the transformation of the nature of physical activity as training increases from slow to intense and remains unchanged for a full three years of training. The main criterion for the division of mesocycles into developing and supporting mesocycles is the total number of sessions in each of them in the selected direction.

The system of mesocycles includes developmental, supportive, and regenerative microcycles that solve their specialized functions. Regarding such stratification of microcycles, it should be noted that in addition to the total number of sessions in the priority orientation, the specialization of the tools used is an additional criterion. 
Planning the process of physical preparation at the different stages of the annual training of 17-19 year old girls on the block method

Experts have found that the development of glycolytic and lactate endurance with the help of specialized tools in football is not always effective, because the achievement of optimal load condition during such exercises is limited by the level of technical and tactical skills of players / 2,3,4,7 /.Therefore, it is advisable to use mainly non-specific means (Ns) as developmental loads in the process of physical training of adolescent girls, and mainly special means (Ms) as supportive loads.

It is known that developmental microcycles can include microcycles that include at least two to three training sessions / 2,3 /.Thus, two and three per week can be considered as full-fledged developers of microcycles, in which they have a predominance of physical activity of a non-specific nature.Microcycles with one or two special training tools per week should be considered as supportive microcycles.Such an approach allows to determine the exact ratio of the use of non-specific and special training tools in microcycles, as well as the dynamics of changes in these ratios within mesocycles, which develop DMSCs and supportive AMSCs throughout the year (Tables 1 and 2).

Table 1: The dynamics of the ratio of DMSCs training tools in developing mesocycles

\begin{tabular}{|c|c|c|c|c|c|c|c|c|c|c|c|c|c|c|}
\hline \multirow{2}{*}{ TB } & \multicolumn{14}{|c|}{ DMSC } \\
\hline & \multicolumn{7}{|c|}{ DMCC } & \multicolumn{7}{|c|}{ AMSC } \\
\hline \multirow{4}{*}{ TB-1 } & 1 & 2 & 3 & 4 & 5 & 6 & 7 & 1 & 2 & 3 & 4 & 5 & 6 & 7 \\
\hline & 8 & 9 & 1 & 11 & 12 & 13 & 14 & 8 & 9 & 1 & 11 & 12 & 13 & 14 \\
\hline & $\mathrm{D}$ & Ns & Ns & Ns & $\mathrm{Tt}$ & $\mathrm{Tt}$ & G & $\mathrm{D}$ & Ms & Ms & $\mathrm{Tt}$ & $\mathrm{Tt}$ & $\mathrm{Tt}$ & G \\
\hline & $\mathrm{D}$ & $\mathrm{Ns}$ & Ns & Ns & $\mathrm{Tt}$ & $\mathrm{Tt}$ & $G$ & $\mathrm{D}$ & Ms & Ms & $\mathrm{Tt}$ & $\mathrm{Tt}$ & $\mathrm{Tt}$ & G \\
\hline all & \multicolumn{7}{|c|}{ Ns-6, Ms-0 } & \multicolumn{7}{|c|}{ Ns-0, Ms-4 } \\
\hline \multirow[t]{3}{*}{ total } & \multicolumn{14}{|c|}{ Number of general development classes:10 } \\
\hline & \multicolumn{14}{|c|}{ Ns-6, Ms - 4 RelativenessNs/Ms - 60:40 } \\
\hline & \multicolumn{7}{|c|}{ DMCC } & \multicolumn{7}{|c|}{ AMSC } \\
\hline \multirow{4}{*}{ TB-2 } & 1 & 2 & 3 & 4 & 5 & 6 & 7 & 1 & 2 & 3 & 4 & 5 & 6 & 7 \\
\hline & 8 & 9 & 1 & 11 & 12 & 13 & 14 & 8 & 9 & 1 & 11 & 12 & 13 & 14 \\
\hline & $\mathrm{D}$ & Ns & Ns & Ns & $\mathrm{Tt}$ & $\mathrm{Tt}$ & $\mathrm{G}$ & $\mathrm{D}$ & Ms & Ms & $\mathrm{Tt}$ & $\mathrm{Tt}$ & $\mathrm{Tt}$ & G \\
\hline & $\mathrm{D}$ & Ns & Ns & Ms & $\mathrm{Tt}$ & $\mathrm{Tt}$ & G & $\mathrm{D}$ & Ms & Ms & $\mathrm{Tt}$ & $\mathrm{Tt}$ & $\mathrm{Tt}$ & G \\
\hline all & \multicolumn{7}{|c|}{ Ns-5, Ms-1 } & \multicolumn{7}{|c|}{ Ns-0, Ms-4 } \\
\hline \multirow[t]{3}{*}{ total } & \multicolumn{14}{|c|}{ Number of general development classes:10 } \\
\hline & \multicolumn{14}{|c|}{ Ns-5, Ms - 5 RelativenessNs/Ms - 50:50 } \\
\hline & \multicolumn{7}{|c|}{ DMCC } & \multicolumn{7}{|c|}{ AMSC } \\
\hline \multirow{4}{*}{ TB-3 } & 1 & 2 & 3 & 4 & 5 & 6 & 7 & 1 & 2 & 3 & 4 & 5 & 6 & 7 \\
\hline & 8 & 9 & 1 & 11 & 12 & 13 & 14 & 8 & 9 & 1 & 11 & 12 & 13 & 14 \\
\hline & $\mathrm{D}$ & Ns & Ns & Ns & $\mathrm{Tt}$ & $\mathrm{Tt}$ & $\mathrm{G}$ & $\mathrm{D}$ & Ms & Ms & $\mathrm{Tt}$ & $\mathrm{Tt}$ & $\mathrm{Tt}$ & G \\
\hline & $\mathrm{D}$ & Ns & Ms & Ms & $\mathrm{Tt}$ & $\mathrm{Tt}$ & G & $\mathrm{D}$ & Ms & Ms & $\mathrm{Tt}$ & $\mathrm{Tt}$ & $\mathrm{Tt}$ & G \\
\hline all & \multicolumn{7}{|c|}{ Ns-4, Ms -2 } & \multicolumn{7}{|c|}{ Ns-0, Ms-4 } \\
\hline \multirow[t]{2}{*}{ total } & \multicolumn{14}{|c|}{ Number of general development classes:10 } \\
\hline & & & & & & Ns-4, & Ms -6 & ti & essN & & & & & \\
\hline
\end{tabular}

Note: DMSCs-developmental mesocycle, TB-training block, DMCCs-developmental microcycle, AMSCs-auxiliary microcycle, Nsmainly non-specific developmental training, Ms-mainly special developmental training, Tt-technical-tactical training; Ggame; $D$ - day off.

As shown in Table 1, the number of sessions in the predominant orientation is not the same in the developing mesocycle, in the DMSCs, in the developmental DMCCs, and in the supporting AMSCs. In the developmental two-week microcycle, such sessions are scheduled three times a week, with a predominance of non-specific loads (Ns).Thus, in a priority-oriented exercise, a "one-way load that performs a specific function of micro ... a deepened development of a certain physical quality" ... emerges (is organized) / 1,2, 4 /.

In turn, two exercises of selective orientation in the predominance of special tools $(\mathrm{Sp})$ in the auxiliary microcycle serve to strengthen the training effect of previous loads in the developmental microcycle. This means that only the proportion of training tools used will change towards specialization when the total number of training sessions on developmental and supportive 
Planning the process of physical preparation at the different stages of the annual training of 17-19 year old girls on the block method

microcycles does not change throughout the year. The noted trend is determined by the guidance on the gradual transformation of training tools from less specialized to more specialized as training increases.

Table 2: Dynamics of the ratio of AMSCs training tools in auxiliary mesocycles

\begin{tabular}{|c|c|c|c|c|c|c|c|c|c|c|c|c|c|c|}
\hline \multirow{2}{*}{ TB } & \multicolumn{14}{|c|}{ AMSC } \\
\hline & \multicolumn{7}{|c|}{ DMCC } & \multicolumn{7}{|c|}{ AMSC } \\
\hline \multirow{4}{*}{ TB-1 } & 1 & 2 & 3 & 4 & 5 & 6 & 7 & 1 & 2 & 3 & 4 & 5 & 6 & 7 \\
\hline & 8 & 9 & 1 & 11 & 12 & 13 & 14 & 8 & 9 & 1 & 11 & 12 & 13 & 14 \\
\hline & $\mathrm{D}$ & Ns & Ns & $\mathrm{Tt}$ & $\mathrm{Tt}$ & $\mathrm{Tt}$ & G & $\mathrm{D}$ & Ms & $\mathrm{Tt}$ & $\mathrm{Tt}$ & $\mathrm{Tt}$ & $\mathrm{Tt}$ & G \\
\hline & $D$ & Ns & Ns & $\mathrm{Tt}$ & $\mathrm{Tt}$ & $\mathrm{Tt}$ & $G$ & $\mathrm{D}$ & $\mathrm{Ms}$ & $\mathrm{Tt}$ & $\mathrm{Tt}$ & $\mathrm{Tt}$ & $\mathrm{Tt}$ & G \\
\hline all & \multicolumn{7}{|c|}{$\mathrm{Ns}-4, \mathrm{Ms}-0$} & \multicolumn{7}{|c|}{ Ns-0, Ms-2 } \\
\hline \multirow[t]{3}{*}{ total } & \multicolumn{14}{|c|}{ Number of general development classes:10 } \\
\hline & \multicolumn{14}{|c|}{ Ns-4, Ms -2 RelativenessNs/Ms - 67:33 } \\
\hline & \multicolumn{7}{|c|}{ DMCC } & \multicolumn{7}{|c|}{ AMSC } \\
\hline \multirow{4}{*}{ TB-2 } & 1 & 2 & 3 & 4 & 5 & 6 & 7 & 1 & 2 & 3 & 4 & 5 & 6 & 7 \\
\hline & 8 & 9 & 1 & 11 & 12 & 13 & 14 & 8 & 9 & 1 & 11 & 12 & 13 & 14 \\
\hline & $\mathrm{D}$ & Ns & Ns & $\mathrm{Tt}$ & Tt & $\mathrm{Tt}$ & G & $\mathrm{D}$ & Ms & $\mathrm{Tt}$ & Tt & Tt & $\mathrm{Tt}$ & G \\
\hline & $\mathrm{D}$ & Ns & Ms & $\mathrm{Tt}$ & Tt & $\mathrm{Tt}$ & G & $\mathrm{D}$ & Ms & $\mathrm{Tt}$ & Tt & Tt & $\mathrm{Tt}$ & G \\
\hline all & \multicolumn{7}{|c|}{ Ns-3, Ms-1 } & \multicolumn{7}{|c|}{ Ns-0, Ms-2 } \\
\hline \multirow[t]{3}{*}{ total } & \multicolumn{14}{|c|}{ Number of general development classes:10 } \\
\hline & \multicolumn{14}{|c|}{ Ns-3, Ms -3 RelativenessNs/Ms - 50:50 } \\
\hline & \multicolumn{7}{|c|}{ DMCC } & \multicolumn{7}{|c|}{ AMSC } \\
\hline \multirow{4}{*}{ TB-3 } & 1 & 2 & 3 & 4 & 5 & 6 & 7 & 1 & 2 & 3 & 4 & 5 & 6 & 7 \\
\hline & 8 & 9 & 1 & 11 & 12 & 13 & 14 & 8 & 9 & 1 & 11 & 12 & 13 & 14 \\
\hline & $\mathrm{D}$ & Ns & Ns & $\mathrm{Tt}$ & $\mathrm{Tt}$ & $\mathrm{Tt}$ & G & $\mathrm{D}$ & Ms & $\mathrm{Tt}$ & $\mathrm{Tt}$ & $\mathrm{Tt}$ & $\mathrm{Tt}$ & G \\
\hline & $\mathrm{D}$ & Ms & Ms & $\mathrm{Tt}$ & $\mathrm{Tt}$ & $\mathrm{Tt}$ & G & $\mathrm{D}$ & Ms & $\mathrm{Tt}$ & $\mathrm{Tt}$ & $\mathrm{Tt}$ & $\mathrm{Tt}$ & G \\
\hline all & \multicolumn{7}{|c|}{ Ns-2, Ms-2 } & \multicolumn{7}{|c|}{ Ns-0, Ms-2 } \\
\hline \multirow[t]{2}{*}{ total } & \multicolumn{14}{|c|}{ Number of general development classes:10 } \\
\hline & \multicolumn{14}{|c|}{ Ns-2, Ms -4 RelativenessNs/Ms - 33:67 } \\
\hline
\end{tabular}

Note: DMSCs-developmental mesocycle, MB-training block, DMCCs-developmental microcycle, AMSCs -Auxiliary microcycle, Ns-mainly non-specific developmental training, Ms- mainly special developmental training, Tt-technical-tactical orientation training; G -game; D-day off.

In contrast to the developmental mesocycle, the number of developmental sessions in the auxiliary mesocycle is reduced, as shown in Table 2, and accordingly the level of exercise impact is also not very high. However, the increase and growth in the share of specialized training tools is maintained.

\section{CONCLUSION}

Thus, the block method of structuring the learning process of adolescent girls allows to systematize the use of training tools, to determine their ratio in the dynamics of the annual training cycle and to clarify the distribution of training in different directions in the annual mesocycles. This implies a transition to a new system of normalization of physical activity. This transition will take the whole process of training girls aged 17-19 to a qualitatively new level.

\section{REFERENCES}

1) Nurimov R.I. Football. Textbook. General edit. under T. 2018. - p. 406.

2) Verxoshanskiy.Yu.V. Theory and methodology of sports training: block system of training of sportsmen of high class / Yu.V. Vexoshanskiy // Theory and practice of physical culture. 2005. -№4. - pp. 24-26.

3) Godik M.A. Physical training of football players in the preparatory period.J. «Fan sportga» 2007 №3. - pp. 18-23.

4) Art of preparation of high-class football players / under ed. N.M. Lyukshinova. - M .: Soviet spot, 2010. - 288 p. 
Planning the process of physical preparation at the different stages of the annual training of 17-19 year old girls on the block method

5) Issuin V.B. Block periodization of sports training: monogaphia / V.B. Issuin. -

6) M .: Soviet sport.. 2010. - 288 p.

7) Mirzabek L.A., Achilov A.M. Methodology for improving the speed-strength training of highly qualified football players. Pedagogical education. 2003, №5. - pp. 48-50.

8) Nistatov E.D. Functional readiness of young football players 15-16 years old in the annual cycle / E.D. Nistratov // theory of practice of physical culture. / - 2000.-№5. - pp. 37-38.

9) Nurimov Z.R. Substantiation of effective means of improving the group tactical actions of qualified football players. Author's abstract. diss. Cand. ped. sciences.T. 2004. - p. 24. 